\title{
Particle-Hole Asymmetry in Doped Mott Insulators: Implications for Tunneling and Photoemission Spectroscopies
}

\author{
Mohit Randeria, ${ }^{1}$ Rajdeep Sensarma, ${ }^{1,2}$ Nandini Trivedi, ${ }^{1}$ and Fu-Chun Zhang ${ }^{3}$ \\ ${ }^{1}$ Physics Department, The Ohio State University, Columbus, Ohio 43210, USA \\ ${ }^{2}$ Tata Institute of Fundamental Research, Mumbai 400 005, India \\ ${ }^{3}$ Physics Department, University of Hong Kong, Hong Kong; Physics Department, University of Cincinnati, \\ Cincinnati, Ohio 45221, USA
}

(Received 7 December 2004; published 19 September 2005)

\begin{abstract}
We use exact sum rules for the one-particle spectral function to quantify the idea that it is more difficult to add an electron than to extract one in a system with strong local repulsion. Our results explain the striking asymmetry in the tunneling spectra of underdoped cuprates which increases with underdoping. We also propose a novel method, based on ratios of sum rules, to estimate local density variations in inhomogeneous materials. Using a variational approach, we show that the origin of the particle-hole asymmetry lies in the incoherent spectral weight.
\end{abstract}

PACS numbers: 74.20.Fg, 74.25.- q, 74.72. $-\mathrm{h}$

With the discovery of high temperature superconductivity in the cuprates there has been enormous interest in doped Mott insulators. One-particle spectroscopies like angle resolved photoemission (ARPES) [1,2] and scanning tunneling microscopy (STM) $[3,4]$ have played a major role in our understanding of these strongly correlated materials. In this Letter we examine in detail sum rule constraints on the single-electron spectral function, focusing, in particular, on the striking asymmetry between occupied and unoccupied spectral weights and its doping dependence in lightly doped Mott insulators. This is in marked contrast with conventional metals and superconductors which exhibit particle-hole (p-h) symmetry on lowenergy scales (tens of meV). Anderson [5] has recently suggested that $\mathrm{p}$-h asymmetry in cuprates may be a characteristic signature of "projection" into a low-energy subspace where strong local Coulomb repulsion makes double occupancy at a site energetically prohibitive.

In this Letter, we make the above idea precise and obtain the following three sets of results. (1) First, we use sum rules for the electron removal (or occupied) and electron injection (or unoccupied) spectral weights in the lowenergy subspace. We show that for the energy-integrated local density of states (LDOS) there is much more weight on the negative bias (occupied) side than on the positive bias (unoccupied) side, and that this asymmetry grows with underdoping. These results quantify the large asymmetry observed in tunneling [6]. We emphasize that these results and the ones described below in (2) are very general, assuming only that the local Coulomb repulsion is the largest energy scale, but otherwise independent of the details of the Hamiltonian and of the nature of the ground state and low-lying excitations. (2) Next, we exploit sum rules to propose a novel way of estimating local charge density variations in inhomogeneous materials from STM data. This is the only known way (that we are aware of) to extract such information. By taking a ratio of local sum rules we eliminate the effect of unknown, spatially varying
STM matrix elements, and find

$$
\frac{\int_{0}^{\Omega_{L}} d \omega g(\mathbf{r} ; \omega)}{\int_{-\infty}^{0} d \omega g(\mathbf{r} ; \omega)}=\frac{2 x(\mathbf{r})}{[1-x(\mathbf{r})]}+\ldots .
$$

where $g(\mathbf{r} ; \omega=e V)$ is the local tunneling conductance and $x(\mathbf{r})$ the local hole concentration at location $\mathbf{r} . \Omega_{L}$ is a cutoff (of order $1 \mathrm{eV}$ in the cuprates) and the omitted terms on the right-hand side represent an order $10 \%$ correction, both of which are discussed in detail below. The ratio on the left-hand side can be estimated from STM data [7], and the above equation can be solved to determine $x(\mathbf{r})$. (3) Finally, we gain further insight into the origin of the p-h asymmetry by decomposing the spectral function into its coherent (quasiparticle) and incoherent pieces for translationally invariant states. For the $d$-wave superconducting state obtained upon doping a Mott insulator [8-10], we explicitly compute the coherent spectral weight using a variational approach for the ground state and low-lying quasiparticle (QP) excitations [10,11]. We thus obtain sum rules for the incoherent spectral weight and find that it dominates the p-h asymmetry. We also find the unexpected result that the incoherent part of the spectral function $A_{\text {inc }}\left(\mathbf{k} ; 0 \leq \omega \leq \Omega_{L}\right)$ is much smaller than the coherent part in this energy range. This is a testable prediction for inverse-photoemission experiments.

Sum rules and asymmetry of local DOS. - We consider a system of electrons described by the Hamiltonian $H=$ $K+U \sum_{i} n_{i \uparrow} n_{i \downarrow}$, where the kinetic energy $K$ describes an arbitrary tight binding dispersion with terms of order $t$ (nearest neighbor hopping). We can also add to $H$ a random one-body potential as well as longer range Coulomb interactions. We will always work in the limit where the local Coulomb repulsion $U$ is much larger than all other energy scales.

The one-electron spectral function $A\left(\mathbf{r}, \mathbf{r}^{\prime} ; \omega\right)=$ $-\operatorname{Im} G\left(\mathbf{r}, \mathbf{r}^{\prime} ; \omega+i 0^{+}\right) / \pi$, where $G$ is the Green's function. We work in real space for two reasons: first, the no- 
double-occupancy constraint is best written in this basis, and second, this allows us to describe spatially inhomogeneous states of lightly doped Mott insulators. We use the $T=0$ spectral representation [12]

$$
\begin{aligned}
A\left(\mathbf{r}, \mathbf{r}^{\prime} ; \omega\right)= & \sum_{m}\left[\left\langle 0\left|c_{\mathbf{r}^{\prime} \sigma}^{\dagger}\right| m\right\rangle\left\langle m\left|c_{\mathbf{r} \sigma}\right| 0\right\rangle \delta\left(\omega+E_{m}-E_{0}\right)\right] \\
& +\sum_{m}\left[\left\langle 0\left|c_{\mathbf{r} \sigma}\right| m\right\rangle\left\langle m\left|c_{\mathbf{r}^{\prime} \sigma}^{\dagger}\right| 0\right\rangle \delta\left(\omega-E_{m}+E_{0}\right)\right],
\end{aligned}
$$

where $|m\rangle$ 's are exact many-body eigenstates with energy $E_{m}$ with $m=0$ the ground state, and $\omega$ is measured with respect to the chemical potential.

The large $U$ suppresses double occupancy at each site, and its effects on the ground state and lowlying excitations are best described using the projection operator $\mathcal{P}=\prod_{r}\left(1-n_{r \uparrow} n_{r \downarrow}\right)$. We then make the well-known unitary transformation [13] $\exp (-i S)$, such that $\exp (i S) H \exp (-i S)$ has no matrix elements connecting states which differ in their double occupancy to any given order in $t / U$. To leading order $i S=-(1 / U) \sum_{\mathbf{r}, \mathbf{r}^{\prime}, \sigma} t_{\mathbf{r r}^{\prime}}\left(n_{\mathbf{r} \bar{\sigma}} c_{\mathbf{r} \sigma}^{\dagger} c_{\mathbf{r}^{\prime} \sigma} h_{\mathbf{r}^{\prime} \bar{\sigma}}-\right.$ h.c. $)+\mathcal{O}(t / U)^{2}$, where $h_{\mathbf{r} \sigma}=1-n_{\mathbf{r}, \sigma}$ and $\bar{\sigma}=-\sigma$. It is useful to incorporate this unitary transformation on the states, which is equivalent to transforming all operators. It then follows that all the low-energy states, i.e., those in the so-called "lower Hubbard band" (LHB), are of the form $\exp (-i S) \mathcal{P}\left|\Phi_{m}\right\rangle$ where $\left|\Phi_{m}\right\rangle$ 's are unprojected states. This characterization of LHB states will be crucial below.

We now derive various exact sum rules without making any assumptions about the ground state or low-lying excitations. For translationally invariant systems these sum rules have been studied earlier $[14,15]$. However, the results are much more general and are particularly useful when applied to a local probe (STM) of spatially inhomogeneous states, as emphasized below.

$\int_{-\infty}^{+\infty} d \omega A\left(\mathbf{r}, \mathbf{r}^{\prime} ; \omega\right)=1$ follows trivially from Eq. (2), while the "occupied" spectral weight [16]

$$
\int_{-\infty}^{0} d \omega A\left(\mathbf{r}, \mathbf{r}^{\prime} ; \omega\right)=\left\langle 0\left|c_{\mathbf{r}^{\prime} \sigma}^{\dagger} c_{\mathbf{r} \sigma}\right| 0\right\rangle .
$$

The LDOS $N(\mathbf{r} ; \omega)=2 A(\mathbf{r}, \mathbf{r} ; \omega)$ (with the two coming from spin) is then given by

$$
\int_{-\infty}^{0} d \omega N(\mathbf{r} ; \omega)=n(\mathbf{r})=1-x(\mathbf{r}) .
$$

Here $n(\mathbf{r})$ is the local electron density and $x(\mathbf{r})$ the local hole doping. This result simply says that there are $(1-x)$ occupied sites (per unit volume) from which one can remove an electron. For a translationally invariant system, we can Fourier transform (3) from $\left(\mathbf{r}-\mathbf{r}^{\prime}\right)$ to $\mathbf{k}$ and obtain the well-known result $\int_{-\infty}^{0} d \omega A(\mathbf{k}, \omega)=n(\mathbf{k})$ which has proved useful in analyzing ARPES data [17].

Next we turn to sum rule constraints on electron addition, probed experimentally by tunneling with a positive bias or by inverse photoemission. It is trivial to derive sum rules for energy integration from 0 to $\infty$ by subtracting the occupied spectral weights (3) or (4) from the total spectral weight of unity. But a much more useful result is obtained by focusing only on the low-energy states in the "lower Hubbard band" (LHB) by integrating over $0 \leq \omega \leq \Omega_{L}$, where the upper cutoff $\Omega_{L}$ satisfies $t \ll \Omega_{L} \ll U$. This is implemented by restricting the sum over intermediate states in (2) to LHB states $|m\rangle=\exp (-i S) P\left|\Phi_{m}\right\rangle$, as discussed above.

We thus get $\int_{0}^{\Omega_{L}} d \omega A\left(\mathbf{r}, \mathbf{r}^{\prime} ; \omega\right)=\sum_{m}\left\langle\Phi_{0}\left|\mathcal{P} \tilde{c}_{\mathbf{r} \sigma} \mathcal{P}\right| \Phi_{m}\right\rangle \times$ $\left\langle\Phi_{m}\left|\mathcal{P} \tilde{c}_{\mathbf{r}^{\prime} \sigma}^{\dagger} \mathcal{P}\right| \Phi_{0}\right\rangle$. Here we found it convenient to use the transformed operator $\tilde{c}_{r \sigma}=\exp (i S) c_{r \sigma} \exp (-i S)$. We now use $\sum_{m}\left|\Phi_{m}\right\rangle\left\langle\Phi_{m}\right|=1$ for unprojected states $\left|\Phi_{m}\right\rangle$ 's, to obtain

$$
\int_{0}^{\Omega_{L}} d \omega A\left(\mathbf{r}, \mathbf{r}^{\prime} ; \omega\right)=\left\langle\Phi_{0}\left|\mathcal{P} \tilde{c}_{\mathbf{r} \sigma} \mathcal{P} \tilde{c}_{\mathbf{r}^{\prime} \sigma}^{\dagger} \mathcal{P}\right| \Phi_{0}\right\rangle .
$$

We next calculate $\mathcal{P} \tilde{c}^{\dagger} \mathcal{P}$ to order $t / U$, and find $\mathcal{P} \tilde{c}_{\mathbf{r} \sigma}^{\dagger} \mathcal{P}=$ $h_{\mathbf{r} \bar{\sigma}} c_{\mathbf{r} \sigma}^{\dagger} \mathcal{P}+\frac{1}{U} \sum_{\mathbf{R}, \sigma^{\prime}} t_{\mathbf{r} \mathbf{R}} h_{\mathbf{R} \bar{\sigma}^{\prime}} c_{\mathbf{R} \sigma^{\prime}}^{\dagger} c_{\mathbf{r} \sigma^{\prime}} n_{\mathbf{r} \bar{\sigma}} c_{\mathbf{r} \sigma}^{\dagger} \mathcal{P}$.

We thus obtain the low-energy sum rule:

$$
\int_{0}^{\Omega_{L}} d \omega N(\mathbf{r} ; \omega)=2 x(\mathbf{r})+2|\langle K(\mathbf{r})\rangle| / U,
$$

where $\quad\langle K(\mathbf{r})\rangle=\left\langle\Phi_{0}\right| \mathcal{P} \sum_{\mathbf{R}, \sigma} t_{\mathbf{R r}}\left(c_{\mathbf{R} \sigma}^{\dagger} c_{\mathbf{r} \sigma}+\right.$ h.c. $) \mathcal{P}\left|\Phi_{0}\right\rangle$. The first term in (6) simply says that one can inject an electron into any of the $x$ empty sites, with the two for spin degeneracy. The second term gives an order $(x t / U)$ correction since the injected electron can create a temporary double occupancy and then hop off to a neighboring empty site. We note that, in contrast to this, the corresponding result (4) to extract an electron is exact to all orders in $t / U$.

We note that the above result for a translationally invariant system $\sum_{\mathbf{k}} \int_{0}^{\Omega_{L}} d \omega A(\mathbf{k}, \omega)=x+|\langle K\rangle| / U$ was derived earlier in Refs. $[14,15]$. We next derive another simple result for the translationally invariant case which we will need later on. First we simplify the right-hand side of (5) using the lowest order expressions for $\mathcal{P} \tilde{c}^{\dagger} \mathcal{P}$ and $\mathcal{P} \tilde{c} \mathcal{P}$. A straightforward calculation then shows that $\int_{0}^{\Omega_{L}} d \omega A\left(\mathbf{r}, \mathbf{r}^{\prime} ; \omega\right)=(1+x) \delta_{\mathbf{r}, \mathbf{r}^{\prime}} / 2-\left\langle c_{\mathbf{r}^{\prime} \sigma}^{\dagger} c_{\mathbf{r} \sigma}\right\rangle+$

$\mathcal{O}(t / U)$. Fourier transforming to $\mathbf{k}$ space and using standard expressions of $n(\mathbf{k})$, we find that the total low-energy spectral weight is

$$
\int_{-\infty}^{\Omega_{L}} d \omega A(\mathbf{k}, \omega)=(1+x) / 2+\mathcal{O}(t / U)
$$

for each $\mathbf{k}$. Note that the deficit from unity comes from weight in the "upper Hubbard band" above $\Omega_{L}$.

Coherent and incoherent spectral weights.-We next decompose the spectral function into its coherent and incoherent pieces and determine their doping dependences. Toward this end we focus on translationally invariant systems and use a variational approach. We take the (variational) ground state to be a projected $d$-wave BCS state 
$|0\rangle=\exp (i S) \mathcal{P}|d \mathrm{BCS}\rangle$ which has given much insight into the phenomenology of the superconducting state of the high $T_{c}$ cuprates [8,9]. Further the (variational) quasiparticle excitations [11] above this ground state are described by $|k \sigma\rangle=\exp (i S) \mathcal{P} \gamma_{k \sigma}^{\dagger}|d \mathrm{BCS}\rangle$, where $\gamma^{\dagger}$ is the standard Bogoliubov QP operator. The QP's lead to the coherent part of $A(\mathbf{k}, \omega)$, i.e., delta functions in $\omega$ at $T=0$, and the relevant matrix elements in Eq. (2) are calculated using the Gutzwiller approximation (GA) [10,11]. Our use of the GA to calculate matrix elements goes beyond previous applications of this approach, which have been by and large restricted to ground state expectation values [18] [see, however, the work of Laughlin [19] where a closely related approximation scheme is used].

We thus obtain [20]

$$
\begin{aligned}
A(\mathbf{k}, \omega)= & Z(\mathbf{k}) u_{\mathbf{k}}^{2} \delta\left(\omega-E_{\mathbf{k}}\right)+Z(\mathbf{k}) v_{\mathbf{k}}^{2} \delta\left(\omega+E_{\mathbf{k}}\right) \\
& +A_{\text {inc }}(\mathbf{k}, \omega),
\end{aligned}
$$

where $u_{\mathbf{k}}, v_{\mathbf{k}}$, and $E_{\mathbf{k}}$ is standard BCS notation [21]. The first two terms in (8) represent the coherent Bogoliubov QP pieces with reduced spectral weight

$$
Z(\mathbf{k})=\frac{2 x}{1+x}+\frac{8 x}{U(1+x)^{2}} \sum_{\mathbf{k}^{\prime}} \epsilon_{\mathbf{k}^{\prime}} v_{\mathbf{k}^{\prime}}^{2}+\frac{4 x}{U(1+x)} \epsilon_{\mathbf{k}} \sum_{\mathbf{k}^{\prime}} v_{\mathbf{k}^{\prime}}^{2}
$$

where $\epsilon_{\mathbf{k}}=2 t\left(\cos k_{x}+\cos k_{y}\right)+\cdots$ is the (negative of the) dispersion corresponding to the bare kinetic energy $K$ in the Hamiltonian. We note that, as emphasized in [9], $Z$ vanishes as one goes to the insulating state at $x=0$, and, in fact, the GA result (9) is in excellent quantitative agreement with the variational Monte Carlo results of Ref. [9]. The sum over all states other than single QP's in (2) leads to the incoherent part of the spectral function denoted by $A_{\text {inc }}(k, \omega)$. Although we cannot calculate its explicit form with the minimal set of assumptions we have made, its existence is necessarily demanded by exact sum rules, as shown below, which also put constraints on $A_{\text {inc }}$.

A nontrivial consistency check of our results is provided by $n(\mathbf{k})$ calculated within GA, which involves only an equal-time ground state correlation and does not depend on any assumptions about QP excited states. We find $n(\mathbf{k})=Z(\mathbf{k}) v_{\mathbf{k}}^{2}+n_{\text {smooth }}(\mathbf{k}) \quad$ where $\quad n_{\text {smooth }}(\mathbf{k})=$ $(1-x)^{2} /[2(1+x)]+\mathcal{O}(t / U)$ is a smooth function of $\mathbf{k}$ in the entire Brillouin zone. We omit the details of the $(t / U)$ corrections which involve rather long expressions [20]. We note that $n(\mathbf{k})$ implies that there is a jump discontinuity along the zone diagonal whose magnitude is given precisely by (9) including the $(t / U)$ corrections.

We now turn to sum rule constraints on $A_{\text {inc }}$ restricting ourselves, for the most part, to leading order results in $t / U$; the next order corrections will be presented elsewhere [20]. We begin by integrating (8) from $-\infty$ to 0 and comparing with the GA result for $n(\mathbf{k})$. We obtain

$$
\int_{-\infty}^{0} d \omega A_{\text {inc }}(\mathbf{k}, \omega)=\frac{(1-x)^{2}}{2(1+x)}+\mathcal{O}(t / U),
$$

which implies that for each $\mathbf{k}$ there is nonzero incoherent spectral weight for $\omega<0$ whose strength, relative to the coherent weight $Z(\mathbf{k}) v_{\mathbf{k}}^{2}$ on the occupied side, increases with underdoping (decreasing $x$ ).

To find the incoherent spectral weight on the unoccupied side we substitute the GA spectral function (8) in the total low-energy spectral weight sum rule (7), leading to $\int_{-\infty}^{\Omega_{L}} d \omega A_{\text {inc }}(\mathbf{k}, \omega)=(1-x)^{2} /[2(1+x)]+\mathcal{O}(t / U)$ for each k. This together with (10) implies that $\int_{0}^{\Omega_{L}} d \omega A_{\text {inc }}(\mathbf{k}, \omega)=\mathcal{O}(x t / U)$. Given the non-negativity of spectral weight, we find that

$$
A_{\text {inc }}\left(\mathbf{k} ; 0 \leq \omega \leq \Omega_{L}\right)=\mathcal{O}(x t / U) .
$$

The vanishing of $A_{\mathrm{inc}}$ for $\omega>0$ to zeroth order in $(t / U)$ is at first sight quite surprising. Although there is very little spectral weight (of order $x$ ) for $\omega>0$, as seen from (6), it is dominated by the coherent piece when $U \gg t$.

To gain more insight into this striking result we derive it in a completely different fashion, which also shows that it is not an artifact of the Gutzwiller approximation. To zeroth order in $(t / U)$ we can set $\exp (i S)=1$, and using the identity $\mathcal{P}_{c_{\mathbf{r}, \sigma}^{\dagger}}^{\dagger} \mathcal{P}=\mathcal{P} c_{\mathbf{r}, \sigma}^{\dagger}$, we find that $\mathcal{P}_{c_{\mathbf{k}, \sigma}}^{\dagger}|0\rangle=$ $\mathcal{P} c_{\mathbf{k}, \sigma}^{\dagger}|d \mathrm{BCS}\rangle=$ const $\times P \gamma_{\mathbf{k}, \sigma}^{\dagger}|d \mathrm{BCS}\rangle=$ const $\times|\mathbf{k}, \sigma\rangle$. We thus find that the projected creation operator acting on the ground state gives precisely the coherent QP state. Thus there is no incoherent weight in the electron creation sector to zeroth order in $(t / U)$.

Finally, one can ask why one does not generate significant incoherent spectral weight through spin rearrangements as one adds an electron to the system. Since such processes involve the superexchange scale $J \sim t^{2} / U$, their contribution to the spectral weight would be of order $x \mathrm{~J} / \mathrm{t}$ which is formally of order $x t / U$, as obtained above.

We can also determine the explicit form of the $\mathcal{O}(t / U)$ incoherent unoccupied spectral weight, which follows from the results described in the paragraph below Eq. (6), together with the GA result [20] for $n(\mathbf{k})$. We find

$$
\sum_{\mathbf{k}} \int_{0}^{\Omega_{L}} d \omega A_{\text {inc }}(\mathbf{k}, \omega)=\frac{2 x(1-x)}{U(1+x)} \sum_{\mathbf{k}} \epsilon_{\mathbf{k}} v_{\mathbf{k}}^{2} .
$$

However, in a $\mathbf{k}$-integrated probe like tunneling it would be hard to separate out the coherent and incoherent contributions, unlike in ARPES where this is possible.

Implications for experiments. - We first discuss the implications of our results for tunneling spectroscopy. The tunneling conductance in STM experiments is proportional to the local density of states $g(\mathbf{r} ; e V)=C(\mathbf{r}) N(\mathbf{r} ; \omega=e V)$ where the $C(\mathbf{r})$ involves $\mathbf{r}$-dependent tunneling matrix elements. Thus our result (6) shows that the (energyintegrated) positive bias conductance is small, of order $x$, while (4) implies that the (integrated) negative bias con- 
ductance is large, of order unity. This provides a qualitative explanation for the large asymmetry seen in STM experiments which show a superconducting gap structure superimposed on a sloping "background" which decreases going from negative to positive bias. Our results predict how this asymmetry should grow with underdoping (decreasing $x$ ). This asymmetry is most strikingly seen in the highly underdoped nonsuperconducting cuprates such as $\mathrm{Na}_{x} \mathrm{Ca}_{1-x} \mathrm{CuO}_{2} \mathrm{Cl}_{2}$ studied by Hanaguri et al. [6]. The nature of the "zero temperature pseudogap state" in such materials is an unsolved problem, and in this context it is very important to reemphasize that our results (4) and (6) make no assumptions about the broken symmetry in the ground state or the nature of low-lying excitations.

To get quantitative information from STM data, we look at ratios in which the unknown tunneling matrix elements cancel out. Taking the ratio of the total unoccupied lowenergy spectral weight (6) to the total occupied spectral weight (4) at the same location $\mathbf{r}$, we obtain

$$
\frac{\int_{0}^{\Omega_{L}} d \omega g(\mathbf{r} ; \omega)}{\int_{-\infty}^{0} d \omega g(\mathbf{r} ; \omega)}=\frac{2 x(\mathbf{r})}{[1-x(\mathbf{r})]}+\frac{2|\langle K(\mathbf{r})\rangle|}{U[1-x(\mathbf{r})]} .
$$

The left-hand side can now be estimated from STM data, provided one can make a reasonable choice of the positive and negative high energy cutoffs [22], and then used to infer the local hole doping $x(\mathbf{r})$ from the first term on the right-hand side of (13). The second term of order $(x t / U)$ gives an estimate of the order $10 \%$ error made in estimating $x$.

In the second part of the Letter we derived results for the doping dependence of the coherent and incoherent parts of the spectral function. The predicted $x$ dependence of the coherent weight $Z$ of Eq. (9) has already been observed in ARPES studies of nodal QP's [see Ref. [9] ]. The important prediction for the very small incoherent spectral weight on the unoccupied side (11) and (12) should be testable in future inverse-photoemission experiments with improved energy resolution.

We have greatly benefited from many stimulating conversations with $\mathrm{P}$. W. Anderson, who first got us interested in this problem. We acknowledge very helpful discussions with J. C. Davis and Y. Kohsaka, and thank them for keeping us abreast of their ongoing experiments. We also thank J.C. Campuzano, O. Fischer, L. H. Greene, P. Hentges, P. A. Lee, P. Phillips, and T.M. Rice for their valuable input. F. C. Z. thanks RGC in Hong Kong for its support.

[1] A. Damascelli, Z. Hussain, and Z. X. Shen, Rev. Mod. Phys. 75, 473 (2003).

[2] J. C. Campuzano, M. R. Norman, and M. Randeria, in Physics of Conventional and Unconventional Superconductors, edited by K. H. Bennemann and J.B.
Ketterson (Springer-Verlag, Berlin, 2004); cond-mat/ 0209476.

[3] Ch. Renner et al., Phys. Rev. Lett. 80, 149 (1998).

[4] S. H. Pan et al., Nature (London) 413, 282 (2001); J.E. Hoffman et al., Science 297, 1148 (2002); K. McKelroy et al., Nature (London) 422, 592 (2003); H. Hanaguri et al., Nature (London) 430, 1001 (2004).

[5] P. W. Anderson (private communication); P. W. Anderson and N. P. Ong, cond-mat/0405518.

[6] See, e.g., the tunneling spectra in K. McKelroy et al. and especially Figs. 1(c) and 3(e) in H. Hanaguri et al. [4].

[7] J. C. Davis and Y. Kohsaka (private communication).

[8] P. W. Anderson, Science 235, 1196 (1987).

[9] A. Paramekanti, M. Randeria, and N. Trivedi, Phys. Rev. Lett. 87, 217002 (2001); M. Randeria, A. Paramekanti, and N. Trivedi, Phys. Rev. B 69, 144509 (2004); A. Paramekanti, M. Randeria, and N. Trivedi, Phys. Rev. B 70, 054504 (2004).

[10] P. W. Anderson, P. A. Lee, M. Randeria, T. M. Rice, N. Trivedi, and F. C. Zhang, J. Phys. Condens. Matter 16, R755 (2004)

[11] F. C. Zhang, C. Gros, T. M. Rice, and H. Shiba, Supercond. Sci. Technol. 1, 36 (1988).

[12] It is straightforward to generalize our results to finite $T$ by introducing the Fermi function in various sum rules.

[13] C. Gros, R. Joynt, and T. M. Rice, Z. Phys. B 68, 425 (1987); A. H. MacDonald, S. M. Girvin, and D. Yoshioka, Phys. Rev. B 37, 9753 (1988).

[14] A. B. Harris and R. V. Lange, Phys. Rev. 157, 295 (1967).

[15] M. B. J. Meinders, H. Eskes, and G. A. Sawatzky, Phys. Rev. B 48, 3916 (1993); H. Eskes and A. M. Oles, Phys. Rev. Lett. 73, 1279 (1994); H. Eskes et al., Phys. Rev. B 50, 17980 (1994).

[16] In Eq. (3) the sum over intermediate states can be simply extended over all states, so that $\sum_{m}|m\rangle\langle m|=1$, even though only states in the LHB actually contribute.

[17] M. Randeria et al., Phys. Rev. Lett. 74, 4951 (1995).

[18] The Gutzwiller approximation results for various ground state observables are in excellent agreement with quantum Monte Carlo results [9]; however, the latter method cannot be used to calculate spectral features.

[19] R. B. Laughlin, cont-mat/0209269.

[20] R. Sensarma, M. Randeria, and N. Trivedi (unpublished).

[21] The dispersion $\xi_{\mathbf{k}}$ entering $u_{\mathbf{k}}, v_{\mathbf{k}}, E_{\mathbf{k}}$ is the sum of a renormalized kinetic energy $2 x \epsilon_{\mathbf{k}} /(1+x)$ and $\mathcal{O}(J)$ Fock shift terms which remain finite as $x \rightarrow 0$. The Fock shifts and the gap are determined by self-consistency equations. The chemical potential is fixed by $2 \sum_{\mathbf{k}} n(\mathbf{k})=N$ which, after using the expression for $n(\mathbf{k})$ described below, reduces to $2 \sum_{\mathbf{k}} v_{\mathbf{k}}^{2}=1-x$. See Ref. [20].

[22] In analyzing experimental data, the cutoff $\Omega_{L}$ should be less than the charge transfer gap $\approx 1.5-2.0 \mathrm{eV}$. On the negative bias side, the ARPES dispersion in Bi2212 shows that the "bottom of the band" is $\approx-0.5 \mathrm{eV}$, but this is only the centroid of a very broad spectral function. Other states begin to show up below about $-1 \mathrm{eV}$ in ARPES $[1,2]$. Thus replacing the " $-\infty$ " by a cutoff $\approx-1.0 \mathrm{eV}$ seems reasonable. 